\title{
The Observed Spin Distributions of Millisecond Radio and X-ray Pulsars
}

\author{
J.W.T. Hessels \\ Astronomical Institute “Anton Pannekoek”, University of Amsterdam, Kruislaan 403, 1098 SJ \\ Amsterdam, The Netherlands; J.W.T.Hessels@uva.nl
}

\begin{abstract}
We consider the currently observed spin distributions of various types of neutron stars, including isolated and binary radio millisecond pulsars in the Galactic plane and globular cluster system as well as neutron stars in low-mass X-ray binary systems where the spin rate is known either through coherent pulsations or burst oscillations. We find that the spin distributions of isolated and binary radio millisecond pulsars are statistically different, at least for those residing in globular clusters, with the binary pulsars being on average faster spinning. This result is likely to hold despite observational biases still affecting the observed spin distribution. A possible explanation for this is that the isolated radio millisecond pulsars are on average older than those in binary systems.
\end{abstract}

Keywords: Neutron Stars; Pulsars; Rotation

PACS: 97.10.Kc,97.60.Gb,97.60.Jd,97.80.-d,97.80.Hn,97.80.Jp

\section{INTRODUCTION}

The number of known $\square^{1}$ neutron stars (NSs) with millisecond spin periods has roughly doubled in the last several years, providing a larger sample with which to consider the spin rate distribution (Table 1). The spin distributions of various sub-classes of NSs should be intimately linked with the nature of the accretion processes in low-mass Xray binary systems (LMXBs) that spin up NSs (i.e. pulsar "recycling") and/or limit their spin-up, such as magnetic torque braking [3, 4, 5] or gravitational wave emission $[6,7,8]$. Thus, a lot of interesting physics is likely to come from mapping these intrinsic distributions and by comparing them to better understand the evolutionary link between NSs LMXBs, presumably the progenitors 2 of the radio millisecond pulsars (MSPs), binary radio MSPs, and isolated MSPs (which are presumably still formed in a binary system, but where the companion star was somehow lost or destroyed).

1 The 61 known radio MSPs in the Galactic plane are from the ANTF catalog [1, http://www.atnf.csiro.au/research/pulsar/psrcat], where we have only selected sources with $B_{\text {surf }}<10^{9} \mathrm{G}$ (with only a few exceptions, this is equivalent to selecting sources with $v_{\text {spin }}>50 \mathrm{~Hz}$ ). The 124 known radio MSPs in GCs with $v_{\text {spin }}>50 \mathrm{~Hz}$ are from the on-line catalog of $\mathrm{P}$. Freire (see http://www.naic.edu/ pfreire/GCpsr.html). The accreting millisecond pulsars are taken from Watts et al. [2], where we have included all 10 known sources with accretion-powered pulsations as well as the 8 sources where the spin rate has only been inferred through the detection of burst oscillations (in at least two separate bursts).

2 Though it may also be that a significant fraction of the MSPs are formed in the accretion-induced collapse of a white dwarf [e.g.9]. 
TABLE 1. Average and median values of spin for various classes of NSs

\begin{tabular}{llll}
\hline Type & Number & $\begin{array}{l}\text { Period }(\mathrm{ms}) \\
\text { mean } / \text { median }\end{array}$ & $\begin{array}{l}\text { Frequency }(\mathrm{Hz}) \\
\text { mean/median }\end{array}$ \\
\hline \hline All MSPs & 185 & $5.0 / 4.4$ & $252 / 227$ \\
Binary MSPs & 117 & $5.0 / 3.8$ & $267 / 262$ \\
Isolated MSPs & 68 & $5.1 / 4.9$ & $225 / 202$ \\
\hline All GC MSPs & 124 & $4.8 / 4.2$ & $258 / 239$ \\
Bin. GC MSPs & 73 & $4.5 / 3.6$ & $288 / 278$ \\
Iso. GC MSPs & 51 & $5.3 / 5.0$ & $215 / 198$ \\
\hline All Field MSPs & 61 & $5.5 / 4.6$ & $239 / 215$ \\
Bin. Field MSPs & 44 & $5.8 / 4.6$ & $233 / 217$ \\
Iso. Field MSPs & 17 & $4.6 / 4.9$ & $253 / 205$ \\
\hline VLMBPs $\left(f\left(m_{1}, m_{2}\right)<10^{-4} \mathrm{M}_{\odot}\right)$ & 21 & $3.3 / 3.5$ & $332 / 287$ \\
LMBPs $\left(f\left(m_{1}, m_{2}\right)>10^{-4} \mathrm{M}_{\odot}\right)$ & 88 & $5.4 / 3.9$ & $254 / 254$ \\
Eclipsing & 22 & $4.4 / 3.5$ & $332 / 282$ \\
Not Eclipsing & 95 & $5.1 / 3.9$ & $252 / 255$ \\
$P_{\text {orb }}<0.5 \mathrm{~d}$ & 37 & $4.5 / 3.6$ & $288 / 274$ \\
$P_{\text {orb }}>0.5 \mathrm{~d}$ & 72 & $5.2 / 3.9$ & $259 / 259$ \\
\hline All LMXBs & 18 & $2.7 / 2.3$ & $436 / 438$ \\
AMXPs & 10 & $3.3 / 2.6$ & $367 / 389$ \\
Burst Osc. & 8 & $2.0 / 1.7$ & $521 / 574$ \\
\hline \hline
\end{tabular}

\section{DISCUSSION OF OBSERVED POPULATIONS}

Radio MSPs: In Figure 1 we plot the spin distribution of all known radio MSPs, both those in the field and in globular cluster (GCs), as well as the accreting NSs in LMXBs where the spin rate is known. The spin distribution of all radio MSPs combined shows a peak around $v_{\text {spin }}=200 \mathrm{~Hz}$, with a sharp decline and tail at higher spin frequencies out to the highest known spin frequency of $716 \mathrm{~Hz}[10]$. The form of the distribution at the highest spin frequencies is likely still strongly affected by observational biases against detecting the fastest-spinning radio pulsars (e.g., due to scattering and binary motion, see also [11] for a detailed discussion of this), whereas the distribution below $200 \mathrm{~Hz}$ is more likely to be intrinsic. In Table 1 we compare the mean and median spin rates of different sub-classes of radio and X-ray MSPs. One of the simplest divisions one can make in the population of radio MSPs is between those with a binary companion and those which are isolated. We find statistical evidence that the spin distributions of binary and isolated radio MSPs are in fact different. A Kolmogorov-Smirnov (KS) test of the two distributions indicates that there is only a $0.03 \%$ chance that the two observed populations are derived from the same instrinsic distribution. Comparing the average and median spin frequencies, we also see that the binary radio MSPs are on average faster-spinning than those that are isolated 3 . One expects observational biases

\footnotetext{
${ }^{3}$ We also note [see also 10], that there is some evidence (though the population of such objects is small) that the fastest-spinning neutron stars are preferentially either in eclipsing systems and/or have a very low
} 


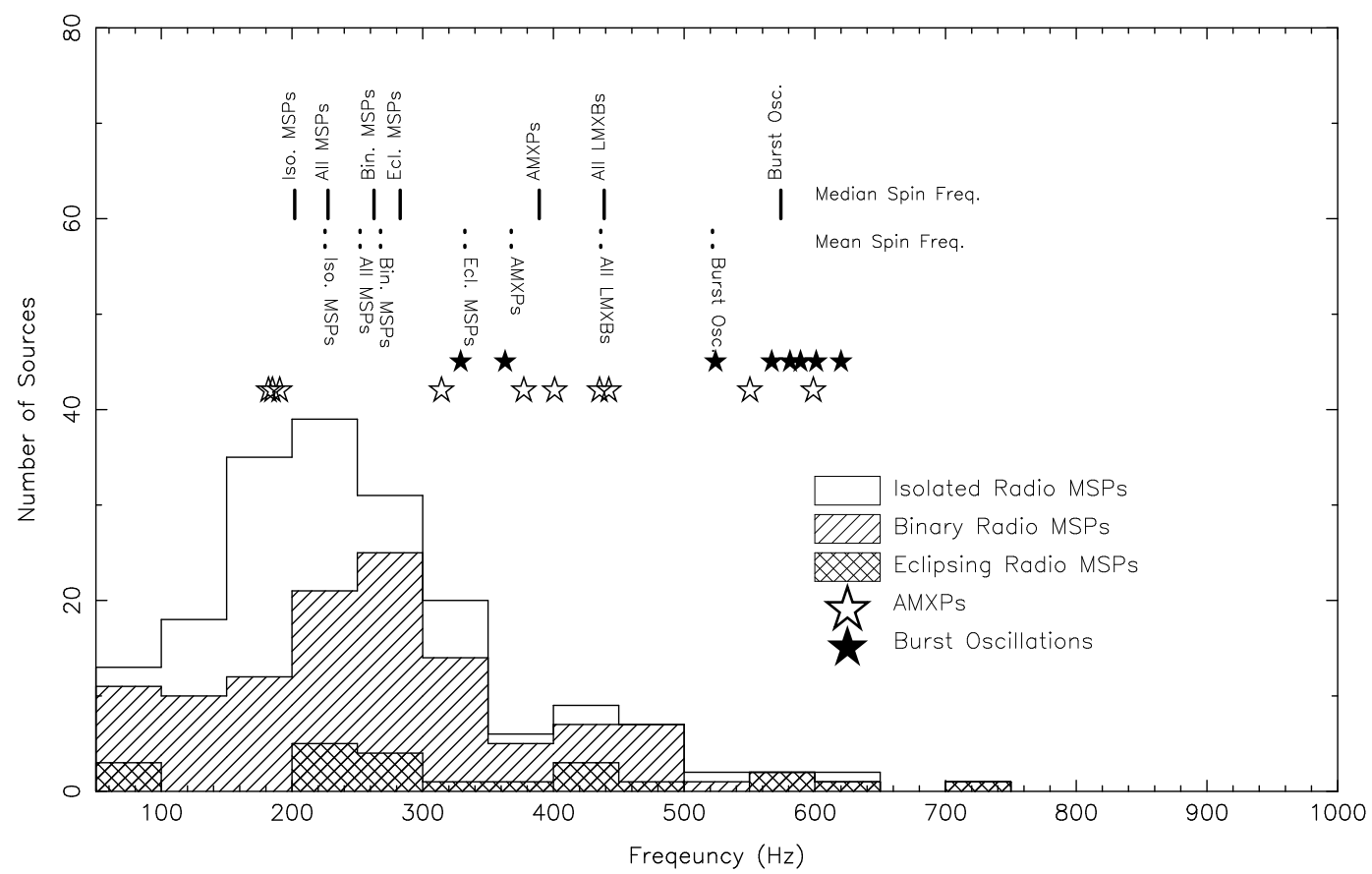

FIGURE 1. The histogram shows the spin frequency distributions of all known radio MSPs (isolated, binary, and eclipsing, including both those in the field and in GCs). The spin frequencies of the 10 known AMXPs and 8 known NMPs are shown above. The average and median spin frequencies of these NS sub-classes are also shown.

still present in the observed distribution to make the binary pulsars appear to spin on average less rapidly than isolated ones. The fact that we observe the opposite scenario implies that, on average, binary MSPs do indeed intrinsically spin faster (note that the luminosity distributions of isolated and binary MSPs, both those in the field and in GCs, appear to be indistinguishable [11, 13]).

However, further sub-dividing the isolated and binary radio MSPs into those found in the Galactic plane versus those found in GCs, we see that there is (as yet) no statistically discernable difference in the spin distributions of isolated and binary MSPs in the Galactic plane [as noted by 13], whereas the spin distribution of binaries and isolated MSPs in GCs are markedly different (KS test indicates $0.0023 \%$ chance that the two observed populations are drawn from the same distribution). The smaller known population of field MSPs, coupled with observational biases, could be responsible for this observed dichotomy. We suspect that the spin distribution of binaries in GCs is possibly less affected by observational bias than that in the Galactic plane because repeated, targetted, deep searches incorporating sophisticated algorithms for detecting binary signals have been made. Indeed, as Table 1 shows, the median spin frequencies of isolated pulsars in the field and in GCs are the same, whereas the binaries in GCs have a much higher median spin frequency than those in the field. We think it is unlikely,

mass companion [the so-called "very low-mass binaries", or VLMBs, see 12]. This could mean that a large fraction of the fastest-spinning radio MSPs are self-obscuring and consequently very hard to detect. 
though this issue warrants further study and consideration, that this difference is due to the dense stellar environments of GCs, which subject a resident NS to potentially several interactions during its life in the cluster core. Thus, we predict that as the population of field MSPs increases, it will become apparent that here too the binary MSPs are on average spinning faster. If this is not found to be the case, an explanation relying on the properties of GC environments will have to be found.

Given their overall similar spin rates, it appears likely that binary and isolated MSPs are formed in a similar process. It still remains possible however that isolated and binary pulsars are formed in somewhat different processes but achieve the same approximate final spin rates because of a common spin limiting mechanism. Here we consider only the former, simpler scenario, and suggest an evolutionary link between them.

A possible, simple explanation for the observed difference in spin rate between the isolated and binary MSPs is that the isolated pulsars are older than those in binaries and have consequently had more time to spin down after they were recycled. Another reason to favour this explanation is that isolated MSPs presumably had binary companions earlier in their lives but have lost or destroyed them over time [e.g. 14]. Using the following expression for spin-down timescale $(\Delta t)$ from initial spin $v_{\circ}$ to final spin $v_{1}$ (assuming moment of inertia $I=10^{45} \mathrm{~g} \mathrm{~cm}^{2}$ and stellar radius $R=10^{6} \mathrm{~cm}$ ):

$$
\frac{1}{v_{1}^{2}}-\frac{1}{v_{\circ}^{2}}=6.15 \times 10^{-7}\left(\frac{B}{10^{8} \mathrm{Gauss}}\right)^{2}\left(\frac{R}{10^{6} \mathrm{~cm}}\right)^{6}\left(\frac{\Delta t}{\mathrm{Gyr}}\right)
$$

we find that it takes 3 Gyr for a pulsar with $B_{\text {surf }}=2.5 \times 10^{8}$ Gauss (the median $B_{\text {surf }}$ of MSPs in the field 4 , where we assume $B_{\text {surf }} \propto \sqrt{P \dot{P}}$ ) to spin down from the median spin frequency of GC binaries $(280 \mathrm{~Hz})$ to the median spin frequency of isolated GC MSPs $(200 \mathrm{~Hz})$. This timescale can be accomodated by the ages of MSPs, which have median characteristic ages $5 \tau_{c}=6 \mathrm{Gyr}$ (defined as $\tau_{c} \equiv P / 2 \dot{P}$ ). We find only weak evidence that the isolated MSPs in the field (median $\tau_{c}=7.2 \mathrm{Gyr}$ ) are significantly older than those in a binary system (median $\tau_{c}=5.6 \mathrm{Gyr}$ ). The two distributions in $\tau_{c}$ are statistically consistent with each other. Again, this could be due to low statistics.

An alternative explanation is that isolated MSPs have, on average, larger magnetic fields than those in binary systems, and consequently spin down on a shorter timescale. Such high- $B_{\text {surf }}$ MSPs would also potentially be more capable of ablating their companion star and becoming isolated. However, comparing the magnetic field distribution of the isolated and binary pulsars in the field, there is as yet no evidence that the distributions of $B_{\text {surf }}$ differ significantly. Again, we will likely have to wait until the known population of field MSPs is significantly larger (or a larger number of GC MSPs have reliable $\dot{P}$ estimates) before we can carefully test this hypothesis.

\footnotetext{
${ }^{4}$ When considering physical parameters that are estimated from spin-down, $\dot{P}$, we will restrict ourselves to those MSPs in the field, who's spin-down, unlike in GCs, is not strongly contaminated by an external gravitational field.

${ }^{5}$ Note however that Ferrario and Wickramasinghe [9] argue that $\tau_{c}$ is 1.5 times the true pulsar age in the case of MSPs
} 
NSs in LMXBs: The population of still accreting NSs with known spin rates is unfortunately still comparatively quite small. However, some interesting differences with the much larger known population of radio MSPs are already apparent [see also 9, 15, for a much more detailed discussion of this]. For instance, the accreting MSPs are on average much faster spinning than the radio MSPs (Table 1). Although such a scenario is potentially over-simplistic [consider 15 , where the link between LMXBs and MSPs is considered from the viewpoint of binary evolution], could the differences in spin rate distributions simply demonstrate the often presumed evolutionary trend from LMXB to binary MSP to isolated MSP, where isolated MSPs are simply older than binary MSPs? In other words, if accretion were to suddenly shut off and these sources became radio pulsars spinning at their current rate, would they naturally evolve into the observed population of radio MSPs (igoring for the time being orbital parameters)? From Eqn. 1 , the implied timescale is $2 \mathrm{Gyr}$ to go from the $440-\mathrm{Hz}$ median spin frequency of the accreting MSPs to the $280-\mathrm{Hz}$ median spin frequency of the binary radio MSPs in GCs. This is not a completely unreasonable timescale, but may be too long to accomodate certain young binary MSPs. This could indicate that further spin-down, in addition to normal magnetic dipole braking, may be necessary. We are planning on investigating this further.

\section{ACKNOWLEDGMENTS}

J.W.T.H. is an NSERC post-doctoral fellow with a supplement from the CSA. We thank Anna Watts, Scott Ransom, Paulo Freire, and Ingrid Stairs for useful discussions and comments.

\section{REFERENCES}

1. R. N. Manchester, G. B. Hobbs, A. Teoh, and M. Hobbs, AJ 129, 1993-2006 (2005).

2. A. Watts, B. Krishnan, L. Bildsten, and B. Schutz, ArXiv e-prints 803 (2008), 0803.4097.

3. P. Ghosh, and F. K. Lamb, ApJ 223, L83-L87 (1978).

4. N. Andersson, K. Glampedakis, B. Haskell, and A. L. Watts, MNRAS 361, 1153-1164 (2005).

5. N. E. White, and W. Zhang, ApJ 490, L87-L90 (1997).

6. J. Papaloizou, and J. E. Pringle, MNRAS 184, 501-508 (1978).

7. R. V. Wagoner, ApJ 278, 345-348 (1984).

8. L. Bildsten, ApJ 501, L89-L92 (1998).

9. L. Ferrario, and D. Wickramasinghe, MNRAS 375, 1009-1016 (2007).

10. J. W. T. Hessels, S. M. Ransom, I. H. Stairs, P. C. C. Freire, V. M. Kaspi, and F. Camilo, Science 311, 1901-1904 (2006).

11. J. W. T. Hessels, S. M. Ransom, I. H. Stairs, V. M. Kaspi, and P. C. C. Freire, ApJ 670, 363-378 (2007).

12. P. C. C. Freire, "Eclipsing Binary Pulsars," in Binary Radio Pulsars, edited by F. A. Rasio, and I. H. Stairs, 2005, vol. 328 of Astronomical Society of the Pacific Conference Series, p. 405.

13. D. R. Lorimer, M. A. McLaughlin, D. J. Champion, and I. H. Stairs, MNRAS 379, 282-288 (2007).

14. V. Radhakrishnan, and C. S. Shukre, Ap\&SS 118, 329-336 (1986).

15. C. J. Deloye, "The Connection Between Low-Mass X-ray Binaries and (Millisecond) Pulsars: A Binary Evolution Perspective," in 40 Years of Pulsars: Millisecond Pulsars, Magnetars and More, edited by C. Bassa, Z. Wang, A. Cumming, and V. M. Kaspi, 2008, vol. 983 of American Institute of Physics Conference Series, pp. 501-509. 